\title{
"P a n i c", a Computer Game for Training of Candidate Physicians Confronted with Mass Casualty Incidents
}

\author{
Nicolas Müller, Patrick Martens,Peter Willen, Henri Müller (-Malek) \\ CUMA, Ruddershove 10, Brugge, Belgium and University of Ghent, Belgium
}

e-mail:nicolas.muller@village.uunet.be,henri.muller@rug.ac.be

Key words: mass casualty management, disaster medicine, on-site computerised decision support system, training device, simulation game

Abstract: $\quad$ Physicians confronted with mass casualty incidents need to switch their mind from the routine on site individual treatment to the exceptional situation of the best overall medical management of group treatment and evacuation to the available hospital facilities. For educational purposes we developed "Panic", a medical decision simulation game. On site real training exercises are critical about used resources, time and costs. Our computerized training module focuses on the joint tasks of triage and regulation. It is an ideal tool to evaluate different physicians' approaches and policy criteria (medical, psychological, organizational and cost) for identical situations. "Panic" can also be used as a pseudo-optimizing decision support system. In that way it is also possible to evaluates the trainees' performances against the experts' protocols and decisions.

\section{BASICS OF DISASTER MEDICINE}

Disaster medicine differs consistently from daily medical practice. Disasters are always unpredicted and unforeseen situations. Their evolution regularly follows the rules of Murphy's law. Dealing with mass casualty incidents remains a challenge for every physician. Managing disasters and 
mass casualty incidents emphasizes the best overall management and treatment of a group. Required resources for treatment and evacuation always exceed the available means.

The aims of disaster medicine are clear and well defined. Every individual patient should receive the best medical treatment on site taking survival chances within the whole patients' population into consideration. The on-site specific problems should not be transferred to the hospitals. Every patient should be treated as soon as possible in the most adequate hospital with regard to his/her injuries. Hospital capacities and treatment possibilities should not be overcrowded.

Unfolding the disaster plan from the medical point of view consists of bringing on site medical teams, technical means and ambulances for the evacuation of the casualties to the hospitals. These plans are activated by the emergency dispatch center covering the disaster area. On the spot three main functions are to be performed by senior medical doctors, specially trained in emergency medicine: the medical director, the triage physician and the regulation physician. The medical director is the overall medical chief on the spot and has to allocate tasks to every arriving medical team. The responsibility of the triage physician consists of examining every casualty and to assign him/her an emergency code using the Mettag card (Fig.1). Every Mettag card has its own identification number and color codes. U1 (red) are patients in need of immediate care. U2 (yellow) are patients who need hospitalization where treatment should be performed within 6 to 8 hours. U3 (green) patients have only minimal injuries, which should be thoroughly examined after all $\mathrm{U} 1$ and $\mathrm{U} 2$ have been treated. U0 (black) are dead or near dead casualties who have no chance of survival. The triage procedure should not last longer than a few minutes per patient and is performed at the entrance of the field hospital (for the process steps, see Fig.1). The regulation physician is the head of the field hospital. He should supervise the start of the adequate treatment and organize the transportation to the most suitable hospital, regarding the urgency type and the injuries. Not all hospital facilities in Belgium are equipped with burn, cardio-thoracic and/or neuro-surgical units. The regulation physician should have good knowledge of all hospitalization facilities in the area. He has a chart containing all the hospitals in the region and their acceptance capacities per hour and per type of urgency (see Annex 2, regulation table). The aim of disaster management in case of mass casualty incidents consists of not overcrowding the surrounding hospital facilities. The organizational efficiency of disaster management consists of bringing the right patient in time to the most suitable hospital regarding his specific injuries.

To avoid chaos on the disasters site and in the hospital facilities two tasks, triage and regulation should be performed in the most suitable way as 
soon as possible after the onset of this specific situation. This needs for all responsible physicians a mental switch from the routine daily individual on site treatment to the best overall group medical treatment and management. Experience learns that this switch is very difficult without adequate training.

\section{PANIC, A COMPUTER PROGRAM USING THE GAME APPROACH}

\subsection{Why developing a game ?}

Coping with disasters remains rare. As managing mass casualty incidents is not a physician's main duty, they are not acquainted and trained for dealing with these exceptional situations. Training in real situations is almost impossible, as a real disaster is not the moment for learning. Physicians should be trained in this specific management long before being confronted with such a kind of incidents. Training possibilities are therefore limited to real situation exercises. Real situation simulations are however critical about the used resources such as ambulances, medical teams and other manpower being taken away from daily emergency service during an exercise. Elementary security rules in case of real incidents within the exercise region could be affected. Real situation simulations are furthermore very expensive and time-consuming. A real on site exercise is also a not reproducible event. Virtual situation simulation such as computer-based gaming seems to be an efficient answer to all the disadvantages of real situation simulations.

\subsection{How the game works...}

The "Panic" game is developed with emphasis on triage and regulation tasks. At the start the following data define the scenario constraints. Casualty descriptions, the available transportation means and the available hospital facilities for evacuation of the disaster site are at the player(s) disposition.

One by one decision combines patient identification number (IDnumber), urgency type, ambulance identification and hospital destination. The choice and the management of the evacuation are done using multiple performance criteria. They endorse medical criteria where the aim is to achieve the shortest throughput time from onset of the incident to the final treatment accommodation or hospital. From the psychological point of view on site waiting times for evacuation should be reduced to a minimum. From the organizational point of view, the time intervals between patients' arrivals in a same hospital facility should be maximal. In order to reduce costs a 
minimal number of ambulances should be involved in the evacuation. Most of these criteria are highly conflicting.

Our computer decision support system contains different databanks. The first one involves all available hospital facilities. For every hospital a given number of patients that can be accepted per urgency type and per hour is defined. These figures (see Annex DSS, second screen shot) are fixed and described in the disaster plans, all approved by the National Health Authorities. Within the U1-urgency type subdivision is made in hospitals with units equipped for the treatment of burned patients, neuro-surgical casualties and/or cardio-thoracic pathologies, as not every hospital can provide these facilities. Besides a databank of hospitals our game has a databank of patients. Every patient chart (see Annex 1, picture 1 and 2) has a description of his/her history with the main clinical findings. Moreover the chart has the hour of arrival in the triage center and an ID-number listed. Some charts even have a picture of the patient. The third databank is one containing all available ambulances with their identification and their on scene time of arrival after the onset of the disaster plan. Time and distance matrices describing the time of a travel loop to the hospital and back to the disaster site including loading (on scene) and unloading (in the emergency department of the hospital) times. These latest times are arbitrary.

\subsection{How the game is played ...}

This game with emphasis on triage and regulation tasks has to be played by one or two trainees. Both triage and regulation tasks can be played separately or combined. The triage task consists of assigning an urgencytype to every casualty entering the triage center. Assigning an urgency-type is associated with possible inter physician variability. The regulation task consists of choosing the right hospital for the right patient on the right time. This decision is made taking urgency-type, hospital capacity and transport abilities into account. The game allows the possibility of overruling hospital capacities to approach real life situations. A patient can however only be dispatched if transportation means are available.

Different regulation policies, using different priority rules can be adopted in coping with the problem. Regarding to patients regulation priority rules, one can choose between the 'overall first come, first served' principle or the 'selective first come, first served' principle, with an absolute priority for U1urgency type casualties. Different hospital selection priority rules can be adopted, choosing f.e. between first to the hospital with the highest overall total capacity, first to the hospital with the highest U1 total capacity, first to the nearest (respective farthest) regional hospitals or first to the nearest 
(respective farthest) hospitals independent of language, national or international boundaries.

\section{THE ADVANTAGES OF USING THIS GAME}

The advantages of using this game are multiple from the scientific and educational point of view. Comparison between different players and the socalled experts can be reviewed and discussed. Same, similar or new scenarios and procedures can be reproduced or created without any problems. Different triage and regulation policies can be tested and evaluated either based on players' performance or created by an automatic expert like simulation program in Arena (see graphics of Annex XIM). Furthermore it is important to get acquainted with the on site use of decision support systems.

The regulation problem and the supporting Panic game turned out to be an useful training tool even outside the medical world, as everyone thinks to be able to master this regulation task without major difficulties. Experience with the virtual disaster planning exercise, Dis-Strat, was gained in different cultural environments. Results proved that this multi-conflicting performance problem is an ideal assignment to show the importance of the need of clear goal definitions and the corresponding explicit quantification of these criteria.

\section{REFERENCES}

Müller N., Muller(-Malek) Decision support system for on-site medical regulation during mass-casualty disasters. H.; Proceedings of the International Congres on computer simulation, ISBN 1-56555-148-6, p366-369

Noto R., Hugenard P. and Larcon A. Médicine de catastrophe. Edition Massaer, ISBN 2-22580885-6

Muller(-Malek) H., Müller N., Martens P., Willen P. Distribution strategy case in 'Cases in Industrial Logistics Management' (ed. Zülch G.) ISBN 3-8265-6369-7, p 185-199

Quarantelli E.L. (1997) Ten criteria for evaluating the management of community disaster Disaster 1997 March; 21(1): p39-56

Chrisie P.M., Levary R.R. (1998) The use of simulation in planning the transportation of patients to hospitals following a disaster. J. Med. Syst. Oct 22(5) pg 289-300

Boer J. (1999) Order in Chaos: modelling medical management in disasters. Eur J Emerg Med June 6(2) pg 141-148

Maniscalco P.M., Christen H.T. (1999) EMS incident management:emergency medical logistics. Emerg Med Serv Jan 28(1) pg 49-52

Levi L., Bregman D., Geva H., Revach M. (1998) Hospital Disaster management simulation systems. Prehospital Disaster Med (1) pg 29-34 


\section{BIOGRAPHY}

\section{Nicolas Müller}

1982 - 1989: medical school RUG

1991: disaster medicine and disaster management

1991 - 1996: anesthesiology and resuscitation

1998: recognized as emergency physician

Since 1998 is he chief flight physician of the CUMA (Center for Urgent Medical Assistance) and staff member of the department of anesthesia and critical care (AZ St. Jan Brugge). He has teaching activities in the university postgraduate program in emergency medicine. And had different lectures on international symposia, referring to the organization of the belgian emergency medical system and prehospital medical care.

\section{Patrick Martens}

1976 - 1983: medical school KUL

1983 - 1988: anesthesiology and resuscitation

1994: $\quad$ recognized Emergency physician

1997: $\quad$ recognized Intensive care Medicine

Since 1990 he is staff member department anesthesia and critical care (AZ St. Jan - Brugge). Since 1997 is Patrick Martens head of the department of Emergency Medicine (AZ St. Jan - Brugge). He has teaching activities in the university postgraduate program in emergency medicine. He is referee of different international scientific journals. He has given different lectures on international symposia, referring to resuscitation and evidence based prehospital care. He is President of the Belgian CPCR group. He is board member of the European Resuscitation Council. Since 1997 is he secretary of the CUMA (Center for Urgent Medical Assistance).

\section{Peter Willen}

He obtained his degree of Civil Engineer in Electro-Mechanics in 1996. $\mathrm{He}$ then graduated as Master in Industrial Management from the Vlerick School for Management. He worked as an assistant to Prof. Muller(-Malek) and Prof. Van Landeghem at the Department of Industrial Management at the University of Ghent. His main research topics were Physical Distribution (Simulation Game), Optimization and Simulation. Since 1999 he works at a consulting company where he specialises in simulation of business processes and supply chains. $\mathrm{He}$ also developed the BPRmodeler ${ }^{\circledR}$, a simulation toolkit for Business Process Simulation.

\section{Henri Muller(-Malek)}

He graduated as a civil engineer from the University of Ghent (Belgium) in 1955, as a mechanical engineer from Delft University (the Netherlands) in 1956, and became faculty associate from the School of Business, Indiana University (USA), in 1957. Since 1962 he taught at the Vlerick School of 
Management, where he was vice president from 1987 to 1992 . He is now an emeritus full professor in Industrial Management at the Universityof Ghent and a visiting professor of several European universities. His interests focuses on business and simulation games for external logistics and decision science support systems. 


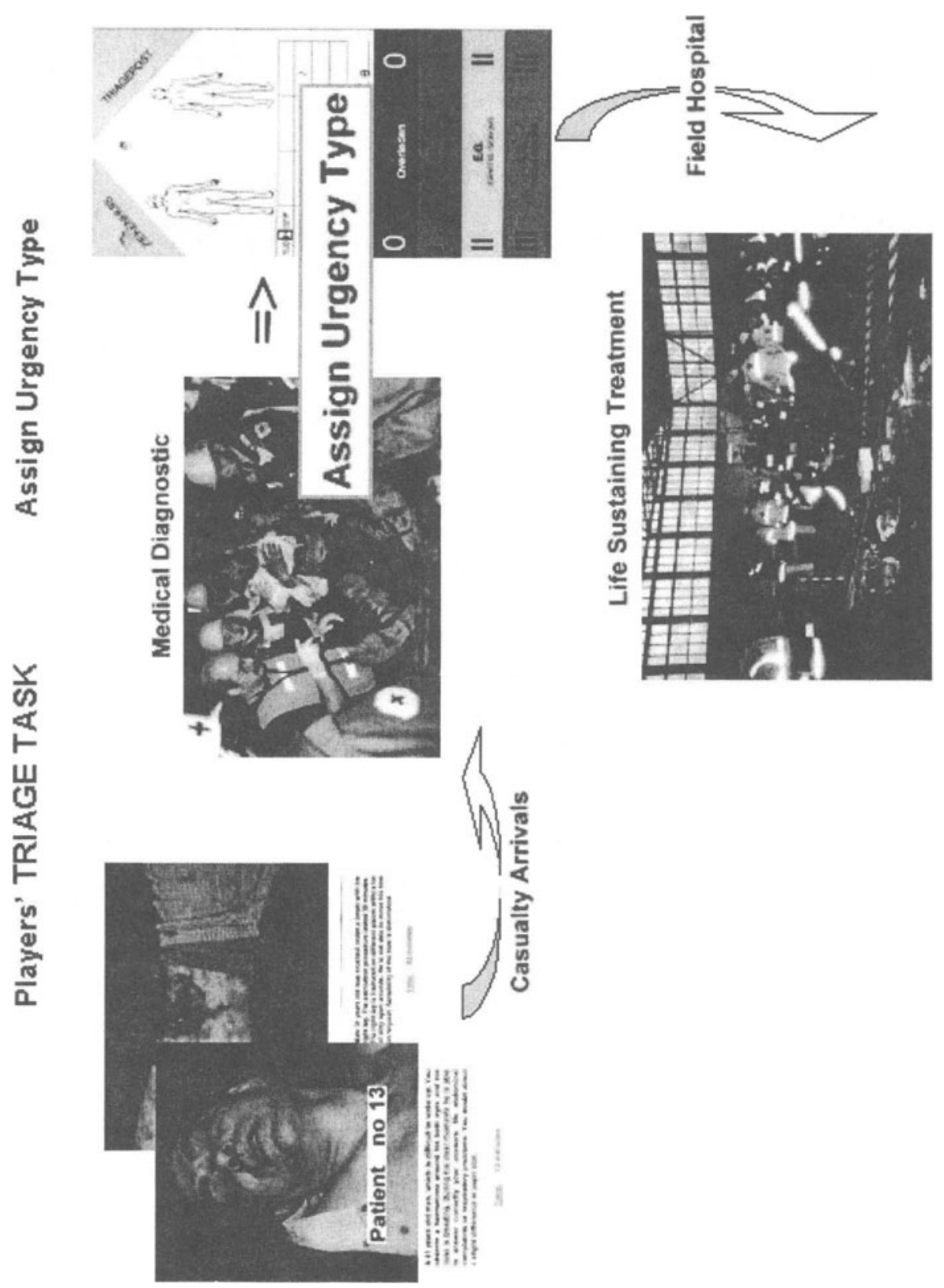

Figure 1. Panic Game 1 


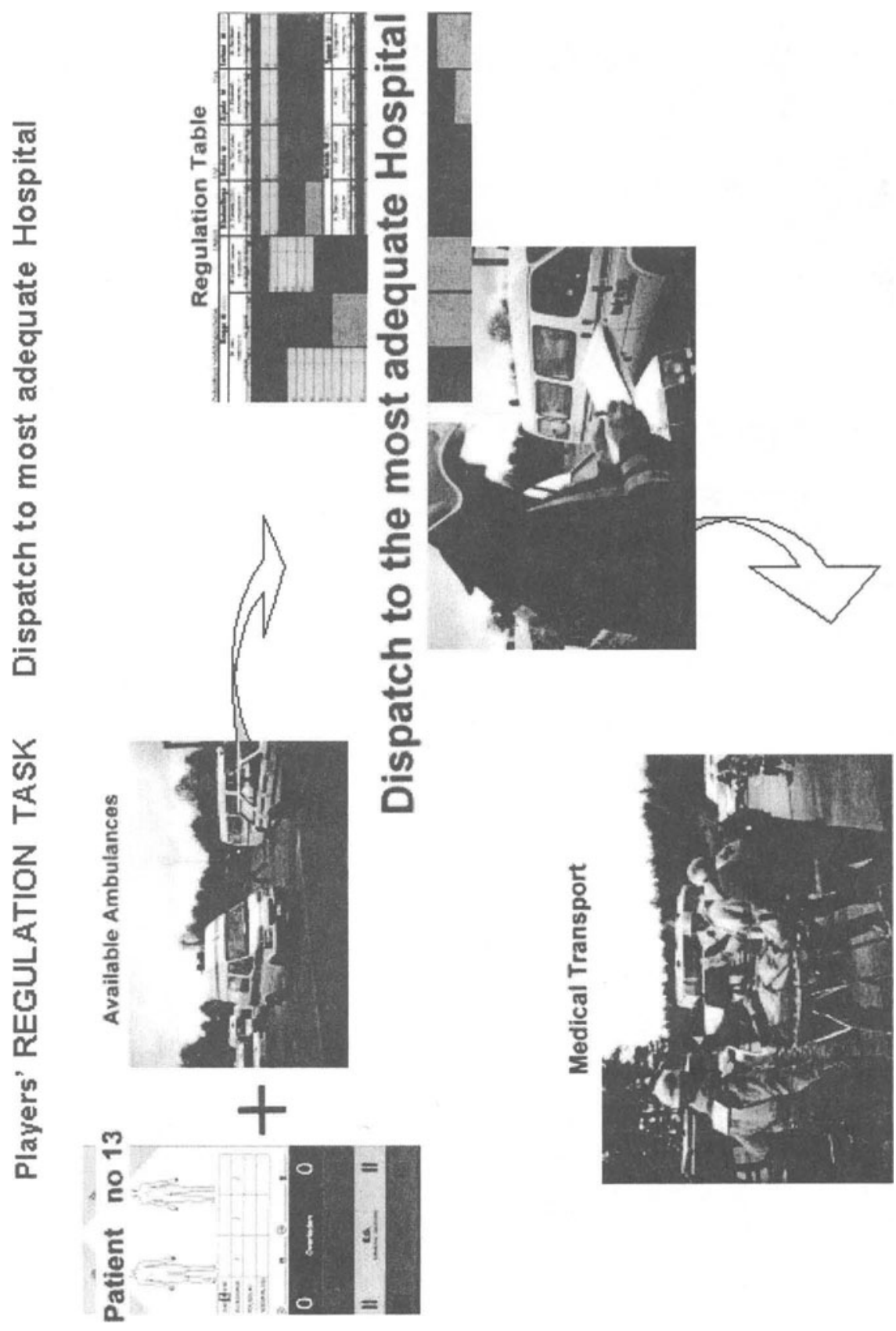

Figure 2. Panic Game 2 


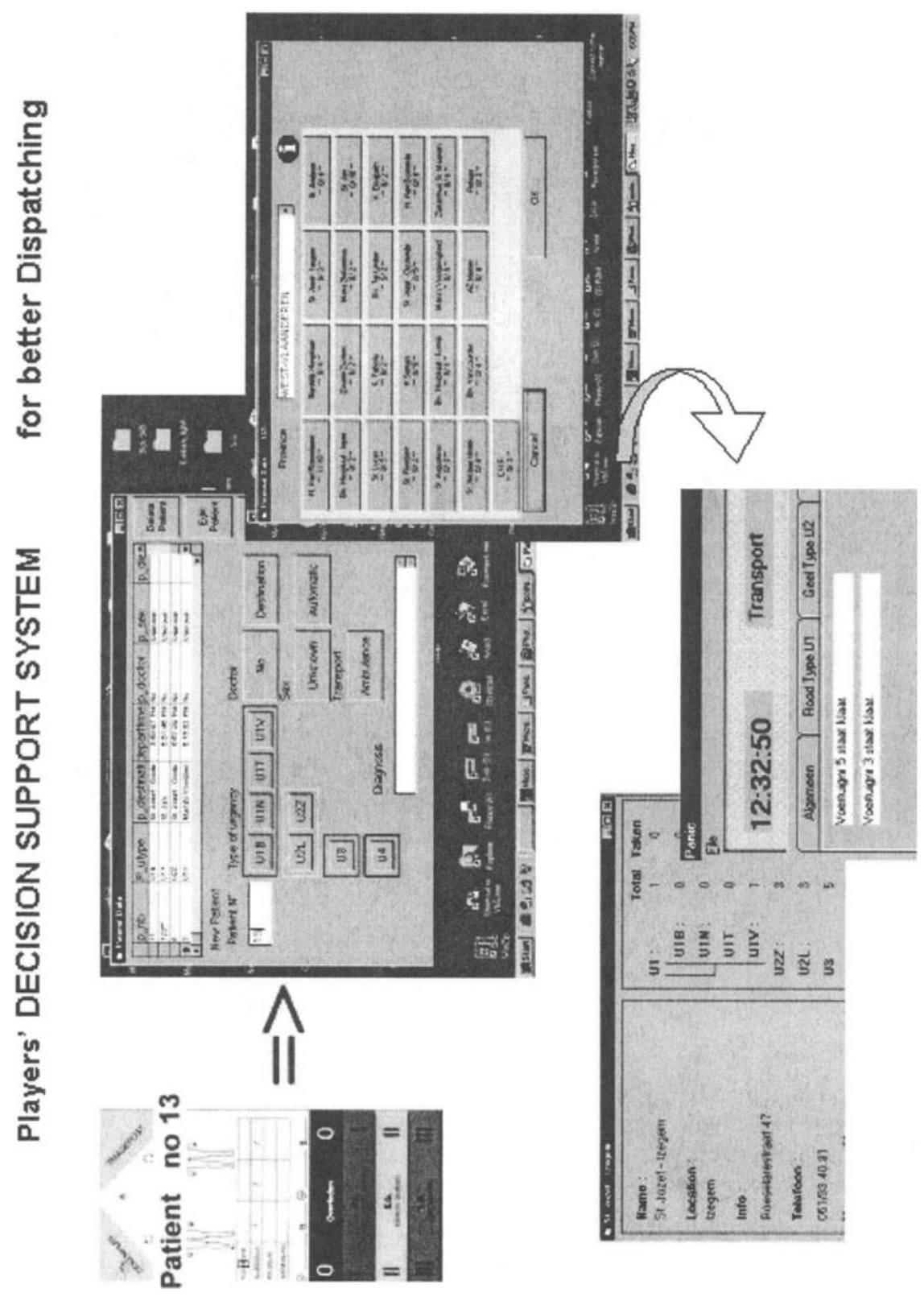

Figure 3. Panic Game DSS 


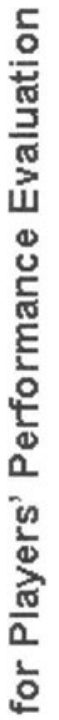

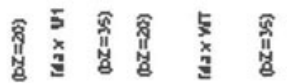
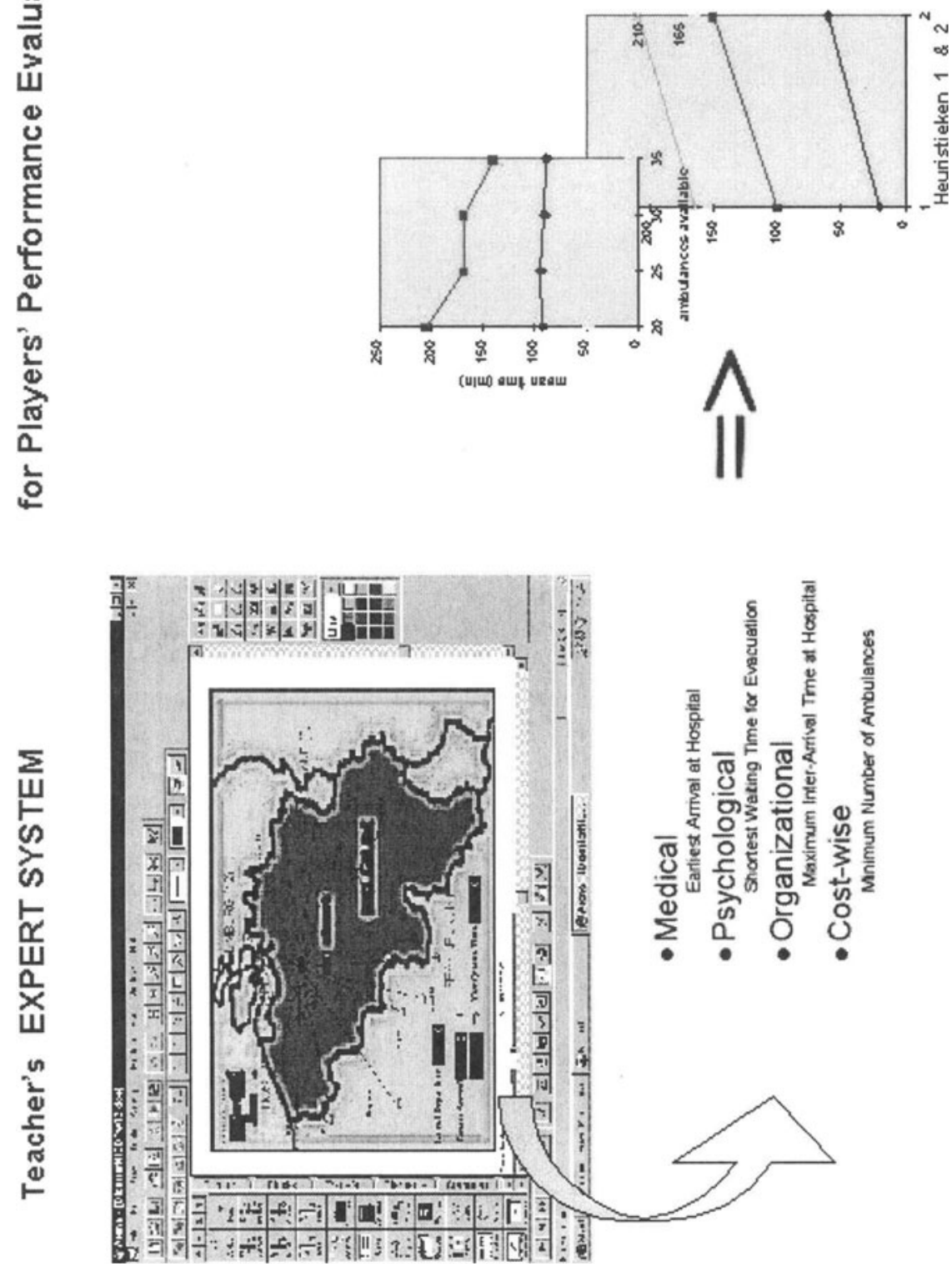

Figure 4. Panic Game XIM 